\title{
A Taxonomy for Denial of Service Attacks in Content-based Publish/Subscribe Systems
}

\author{
Alex Wun \\ wun@eecg.utoronto.ca \\ Alex Cheung ${ }^{\dagger}$ \\ Hans-Arno Jacobsen \\ cheung@eecg.utoronto.ca jacobsen@eecg.utoronto.ca \\ $\dagger$ Department of Electrical and Computer Engineering \\ $\ddagger$ Department of Computer Science \\ University of Toronto
}

\begin{abstract}
Denial of Service (DoS) attacks continue to affect the availability of critical systems on the Internet. The existing DoS problem is enough to merit significant research dedicated to analyzing and classifying DoS attacks in the Internet context. However, no such research exists for DoS attacks in the domain of Content-based Publish/Subscribe (CPS) systems despite CPS being at the forefront of business process execution, application integration, and event processing applications. This can be attributed to the lack of structure and understanding of key issues in the area of DoS in CPS systems. In this paper, we propose to address these problems by presenting a taxonomy for classifying DoS characteristics and concerns new to CPS systems. Our taxonomy is motivated by a number of experimental results that were obtained using our CPS middleware implementation and that highlight fundamental DoS concerns in this domain. Finally, we discuss some example DoS attacks in detail with respect to our taxonomy and experimental results. We find that localization, message content complexity, and filter statefulness are the key CPS characteristics to consider when designing DoS resilient CPS systems.
\end{abstract}

\section{Categories and Subject Descriptors}

H.4.m [Information Systems Applications]: Miscellaneous

\section{General Terms}

Security, Reliability

\section{Keywords}

Publish/Subscribe, Denial of Service, Security

\section{INTRODUCTION}

Denial of Service (DoS) attacks remain disturbingly prominent in the Internet despite continuous defensive efforts.

Permission to make digital or hard copies of all or part of this work for personal or classroom use is granted without fee provided that copies are not made or distributed for profit or commercial advantage and that copies bear this notice and the full citation on the first page. To copy otherwise, to republish, to post on servers or to redistribute to lists, requires prior specific permission and/or a fee.

DEBS '07, June 20-22, 2007 Toronto, Ontario, Canada

Copyright 2007 ACM 978-1-59593-665-3/07/03 ...\$10.00.
This is primarily because the Internet was designed for efficient packet forwarding rather than for security. Due to the Internet's design, an ongoing issue persists in which supplementary security solutions are typically used to compensate for the lack of secure networking services and to protect against malicious attacks. As a consequence of this ongoing issue, organizations are reluctant to deploy new technologies that are not either secure by design or compatible with existing security frameworks. As a novel technology, Content-based Publish/Subscribe (CPS) systems are poised to provide expressive, loosely-coupled, event-based messaging services in many enterprise applications. In particular, CPS and related technologies are at the forefront of business process execution, application integration, and event processing networks $[1,2]$. But despite the many advances made in CPS research and the importance of CPS technology, adoption will be slow unless the DoS resilience characteristics of this new paradigm are well understood. A major step towards this goal is to identify DoS vulnerabilities new to CPS systems and how CPS systems are impacted by such vulnerabilities. As part of this step, we propose a taxonomy for classifying DoS attacks in CPS systems. We hope that structuring the domain of DoS attacks will facilitate further research and discussion in the area.

Motivating our proposed taxonomy are several experimental results that illustrate some fundamental DoS concerns that are new to CPS systems. In particular, we find that some CPS entities are inherently more resilient to DoS attacks due to localization effects, a measure of message complexity will be needed to maintain control over performance, and the stateful nature of CPS systems has cumulative effects on DoS attacks. These new concerns imply new techniques for achieving DoS attack detection, prevention, and recovery if CPS systems are to be highly available.

Finally, we also include detailed discussions of specific DoS attack instances to provide further insight into the implications of CPS state on DoS vulnerabilities.

The contributions of this paper are: (1) Experimental results that highlight fundamental DoS concerns new to CPS systems, (2) A taxonomy for classifying DoS attacks unique to CPS systems, and (3) a discussion of specific DoS attack instances providing insight to CPS vulnerabilities and the importance of a taxonomy.

The remaining sections of this paper are organized as follows: Section 2 provides background information on CPS systems, Section 3 presents our experimental analysis, Section 4 presents our proposed taxonomy, Section 5 presents a discussion of specific DoS attacks with respect to our taxon- 
omy and experimental results, Section 6 discusses the related work, and Section 7 is our conclusion.

\section{BACKGROUND}

Publish/Subscribe is an event-based messaging model in which event notifications are delivered to clients based on their prior expressed interests. Content-based Publish/Subscribe (CPS) systems in particular, allow clients to express fine-grained interest using filters (subscriptions) consisting of predicates over potential publication content.

CPS systems generally distinguish between brokers and clients. Brokers are typically the entities that perform message matching and forwarding while clients issue publication and subscription messages (advertisement messages as well to define event schema when applicable). With respect to a certain event schema (an advertisement tree or message type for example), we can further distinguish edge brokers from internal brokers as well as publisher clients from subscriber clients.

Edge brokers are brokers hosting clients that publish or subscribe to an event schema while internal brokers only participate in matching or routing messages. That is, edge brokers are the proxy through which clients issue messages. In Figure $6(\mathrm{a})$, brokers $B_{S}$ and $B_{P}$ are edge brokers while broker $B_{0}$ is an internal broker.

Publisher and subscriber clients are those that exclusively issue publications and subscriptions, respectively. Typically however, a single client will likely take on both publisher and subscriber roles (possibly to different event schemas).

\section{DENIAL OF SERVICE EFFECTS}

With the goal of developing a taxonomy, we identified several important CPS system characteristics based on our research experience. To investigate the DoS implications of these characteristics, we performed experiments using a general-purpose, Java-based CPS middleware platform developed by our research group ${ }^{1}$. In all experiments, each broker ran on a dedicated Dual-Core Intel Xeon system running Linux with $2 \mathrm{~GB}$ of memory. DoS workloads were produced by clients also running on their own dedicated system. The results from these experiments support the DoS effects described and classified in our taxonomy. Specifically, the results highlight new DoS concerns that arise in CPS systems due to the use of distributed brokerage, content-based routing, and stateful message filtering. It is important to note that although the experiments were performed using our CPS middleware implementation, the results are based on concepts applicable to CPS systems in general. More specifically, regardless of the actual algorithms used in our implementation, the matching times in our experiments are comparable to the millisecond ranges reported by other CPS implementations in the literature $[4,9]$.

\subsection{Localization Effects}

In a distributed CPS system, the effects of a DoS attack will propagate differently depending on factors such as message content and volume. In particular, heavy workloads tend to create bottlenecks in the system that interfere with message propagation. We performed some experiments to illustrate this fundamental point with three brokers. In our

\footnotetext{
${ }^{1}$ http://padres.msrg.utoronto.ca
}

setup, there are two edge brokers and one internal broker between the edge brokers. Publication-based attack workload streams are injected at Broker $\mathrm{A}$ and subscribed to from Broker C. Each stream injects 10-tuple publications at a rate of 100 publications per second and we gradually add publication streams as time progresses. At each broker, we use another publication stream to measure the response time of delivering notifications at that broker. Although the same attack workload passes through all three brokers, Figure 1(a) shows that the response times at each broker differ significantly. The response times at Broker A (the broker being directly targeted by the publication flood) quickly grow to unacceptable levels under heavy DoS load. However, internal Broker B maintains good response times. Broker $\mathrm{C}$ suffers from unacceptable response times as well despite not being directly targeted by the publication flood. In fact, Broker $\mathrm{C}$ shows even worse response times than Broker A. This phenomenon is explained in Figure 1(c), which shows the input and output queueing delays at each broker. Since matching delays were insignificant at all three brokers, plots for the matching delays have been omitted. Internal Broker $\mathrm{B}$ experiences low delays while Broker A experiences very high input queueing delays due to the direct flood of publications. Broker $\mathrm{C}$ suffers from both high input and output queueing delays due to the extra processing required to deliver notifications to each stream subscriber. Note that the processing delays are plotted using a log scale y-axis. If Broker $\mathrm{C}$ does not host a large number of subscribers, then its processing delays are comparable to that of Broker B. Figure 1(b) further shows that the internal broker is the least loaded out of the three brokers, both in terms of system memory and input queueing ${ }^{2}$.

This experiment illustrates two key characteristics of DoS attacks that are new in the area of CPS systems. First, heavy load causes bottlenecks in the system that can prevent effective propagation of DoS attacks. In this case, internal brokers do not feel the impact of the publication flooding attack. We refer to this as localization of a DoS attack's effects. Second, it is possible for an attack to induce effects at distant brokers without affecting all brokers along the path of propagation. We refer to this as transmission of a DoS attack's effects. In this experiment, the effects of the DoS attack are transmitted to Broker C by the workload source without affecting Broker B.

The first characteristic is a benefit of distributed CPS systems, since DoS counter-measures can take advantage of localization to achieve high availability. However, the second characteristic is something that systems must carefully guard against since transmission enables dangerous remote attacks from potentially arbitrary points in the network. For instance, an adversary can conceivably craft a malicious stream of publications to match all subscriber interests at some remote broker. As we show, this malicious traffic does not necessarily affect intermediate brokers en route to the remotely targeted broker. Consequently, early detection of transmitted DoS attacks is much more difficult.

\subsection{Workload Complexity Effects}

In contrast to the fixed packet formats used in lowerlevel network layers, CPS messages are highly flexible in content. However, the key benefit of flexible and expres-

${ }^{2}$ Note that the input queue size has been scaled down for graphing with free memory against the same axes. 


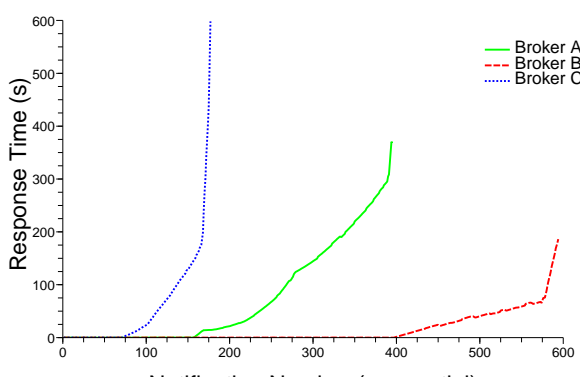

Notification Number (sequential)
(a) Response Times

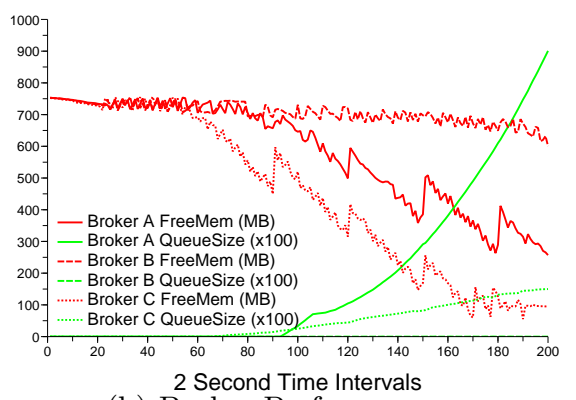

(b) Broker Performance

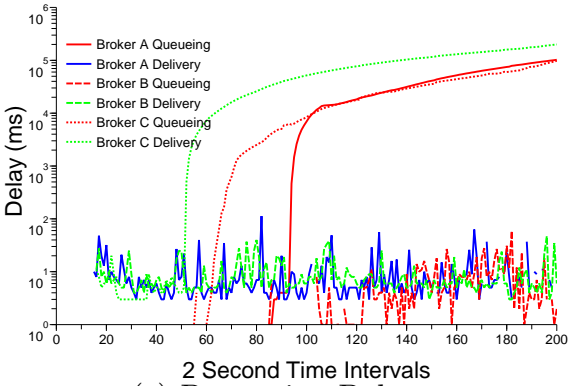

(c) Processing Delays

Figure 1: Localization of DoS effects in distributed CPS systems.

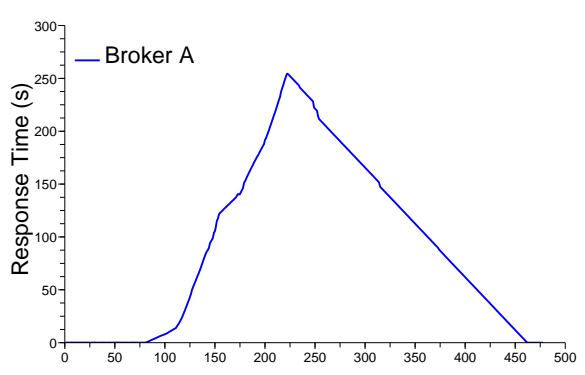

Notification Number (sequential)
(a) Response Time

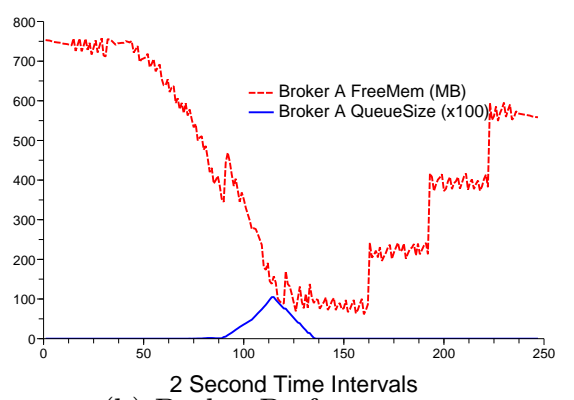

(b) Broker Performance

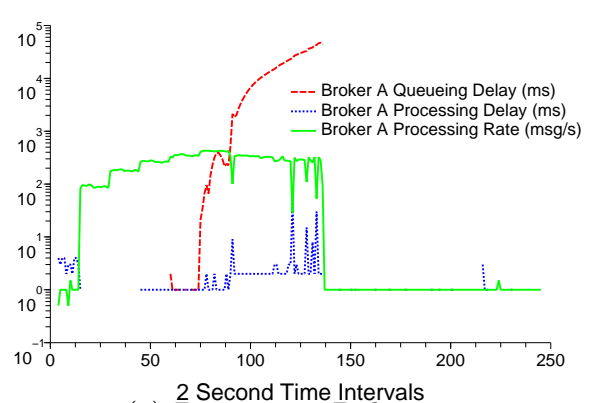

(c) 2 Second Time Intervals

Figure 2: System recovery characteristics under low complexity DoS attack workloads.

sive messaging also leads to DoS concerns since processing requirements can vary wildly from one attack workload to another. Message complexity will significantly affect how a system recovers from DoS attacks and includes characteristics such as message size, the types of filtering operations involved, and message structure (e.g., number of predicates, integer/string comparisons versus arbitrary Boolean functions, and attribute/value pairs versus XML, respectively). We performed some experiments to illustrate the fundamental effects of message complexity in a DoS attack. In our setup, a single broker is loaded with streams publishing at a rate of 100 publications per second. We gradually add streams as time progresses to overload the broker and then stop all DoS traffic to observe how the system recovers. Figures 2(b) and 3(b) show the memory consumption and input queue sizes of the broker for low complexity (10-tuple) and high complexity (100-tuple) attack workloads, respectively. Clearly, higher complexity messages require more processing and cause the broker to become overloaded faster and recover more slowly. Figures 2(c) and 3(c) show the processing delays for low and high complexity attack workloads, respectively. As expected, we can see that processing delays are greater under high complexity attack workloads, leading to lower processing rates. Consequently, the processing of malicious messages through the broker drastically slows down the recovery of queueing delay. In this experiment, the same stream of publications used in the propagation experiments to measure response times were used here as well. Figures 2(a) and 3(a) show the response times under low and high complexity message workloads, respectively. As expected, the response times are much worse and take longer to recover when the broker is being attacked with high complexity message floods. An important observation is that the peaks in the response time graphs do not correspond to the input queue peaks in Figure 2. Response times do not begin recovering immediately after the DoS attack stops because malicious messages are queued up and continue to load the broker. In fact, response times do not start recovering until queueing delays begin to recover. Consequently, response times at the broker continue to become worse even as queued messages are processed through the broker. In Figure 2(a), notification message \#150 corresponds to the point in time when the DoS attack stops. At this point, there is a noticeable change in the response time curve. In Figure 3(a), notification message \#60 corresponds to this same point. Notice that the attack based on high complexity messages caused response times to continue growing for much longer after the actual attack has ended.

This experiment demonstrates that the performance of CPS systems can vary significantly with workload complexity. This implies that DoS resilient systems must have some measure of message and workload complexity in order to maintain predictable control over performance and response times. In current systems, tuple and predicate counts are likely a reliable measure of complexity. However, as filtering expressiveness becomes more sophisticated and moves beyond numeric and string comparisons, the cost of performing any given filtering operation will have a significant impact as well.

\subsection{Message State Effects}

CPS systems must necessarily maintain state for perform- 


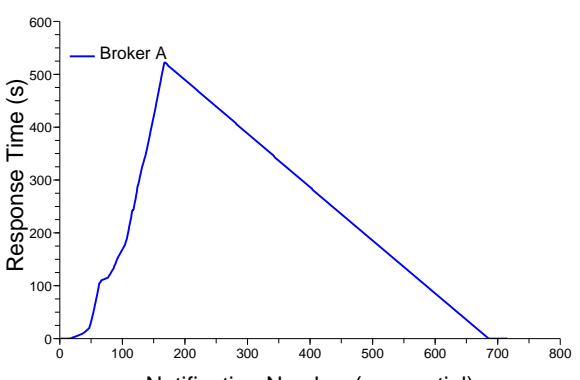

Notification Number (sequentia)
(a) Response Time

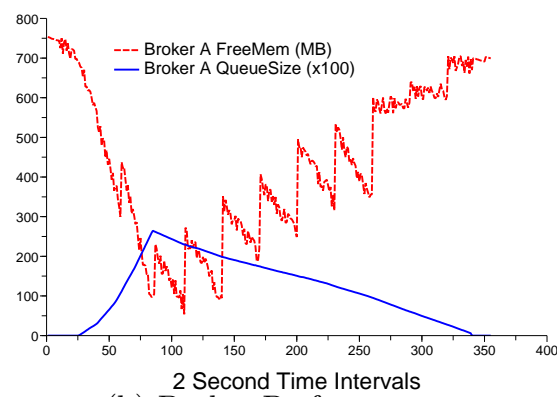

(b) Broker Performance

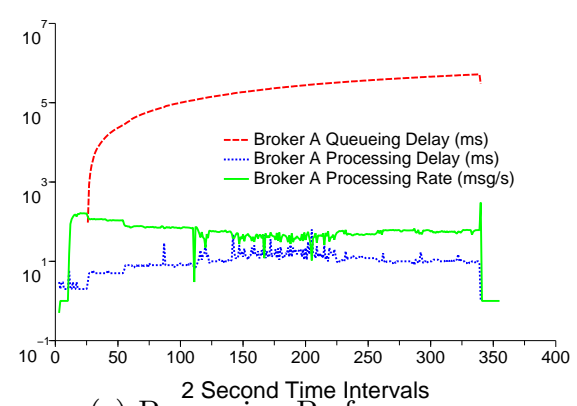

(c) Processing Performance

Figure 3: System recovery characteristics under high complexity DoS attack workloads.

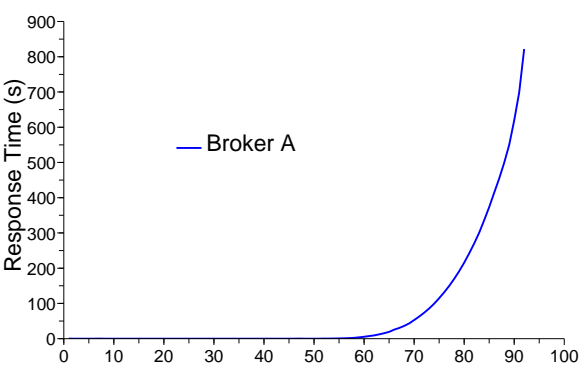

Notification Number (sequential)

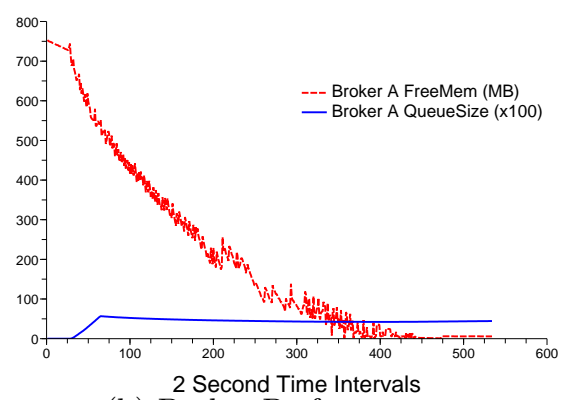

(b) Broker Performance

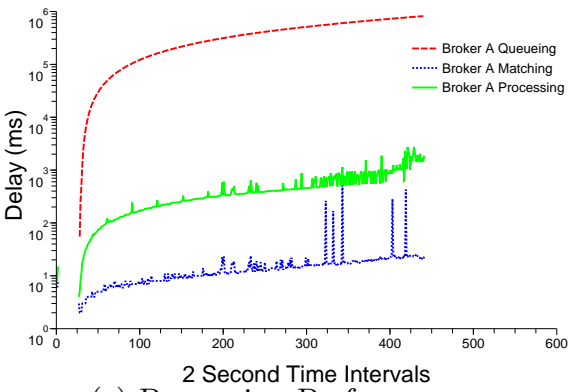

(c) Processing Performance

Figure 4: System recovery characteristics under stateful DoS attack workloads.

ing publication filtering or event correlation. State allows CPS systems to provide powerful event-driven services, but also opens up new avenues of attack for adversaries. In this experiment, we demonstrate the fundamental severity of stateful DoS attacks. The setup and experimental approach are the same as used in the workload complexity experiments. However, we now use malicious workload streams consisting of low complexity (10-predicate) subscriptions rather than publications. The rate also remains the same at 100 subscriptions per second. Figure 4(b) shows the effects of this subscription flooding attack on the broker. Comparing this subscription based attack to the publication based attack in Figure 2(b), we see a significant difference despite the equivalent message sizes and workload message rates. The increased severity of the attack results from additional state maintenance during subscription insertions. Most importantly, the effects of this attack are cumulative since each inserted subscription impacts the processing of subsequent subscriptions. In fact, Figure 4(c) shows that the processing time for inserting subscriptions into the matching engine data structure alone increases almost an order of magnitude over the course of the experiment. Overall processing time for subscriptions increases almost two orders of magnitude. As a result, the rate of processing messages from the queue becomes similar to the rate of incoming publications used to measure response time. This is reflected by the nearly flat input queue size in Figure 4(b). In this particular experiment, the broker was unable to recover and eventually ran out of memory. Figure 4(a) shows how the corresponding response time grows exponentially and never recovers. This experiment demonstrates the impact of stateful DoS attacks caused by state management overheads and the cumulative effects of message state. This is a severe problem for CPS systems which target large-scale applications involving millions of subscribers. Often, state management techniques such as message expiry, event lifetimes, and message consumption policies are used to manage exploding state. However, these techniques manage legitimate state and will likely fail under malicious stateful attacks. DoS resilient systems will need mechanisms to control and manage malicious state which may require the development of intrusion detection techniques unique to CPS systems.

\section{TAXONOMY SUMMARY}

Motivated by the results of our DoS experiments, our taxonomy provides a coarse-grained summary of important DoS attack characteristics in the CPS domain. This classification reflects both entirely novel attack characteristics and new considerations for existing attack characteristics in this domain. To the best of our knowledge, there have not been any previous efforts to come up with a classification scheme for DoS attacks in CPS systems. Each class we propose is important because different defence mechanisms will be required depending on the classification of the attack. The classes used in our taxonomy are also independent of one another so that an attack can be characterized under one or more classes. Our taxonomy is summarized graphically in Figure 5 and applied to a few examples attacks in Section 5.

\subsection{Exploitation Type}




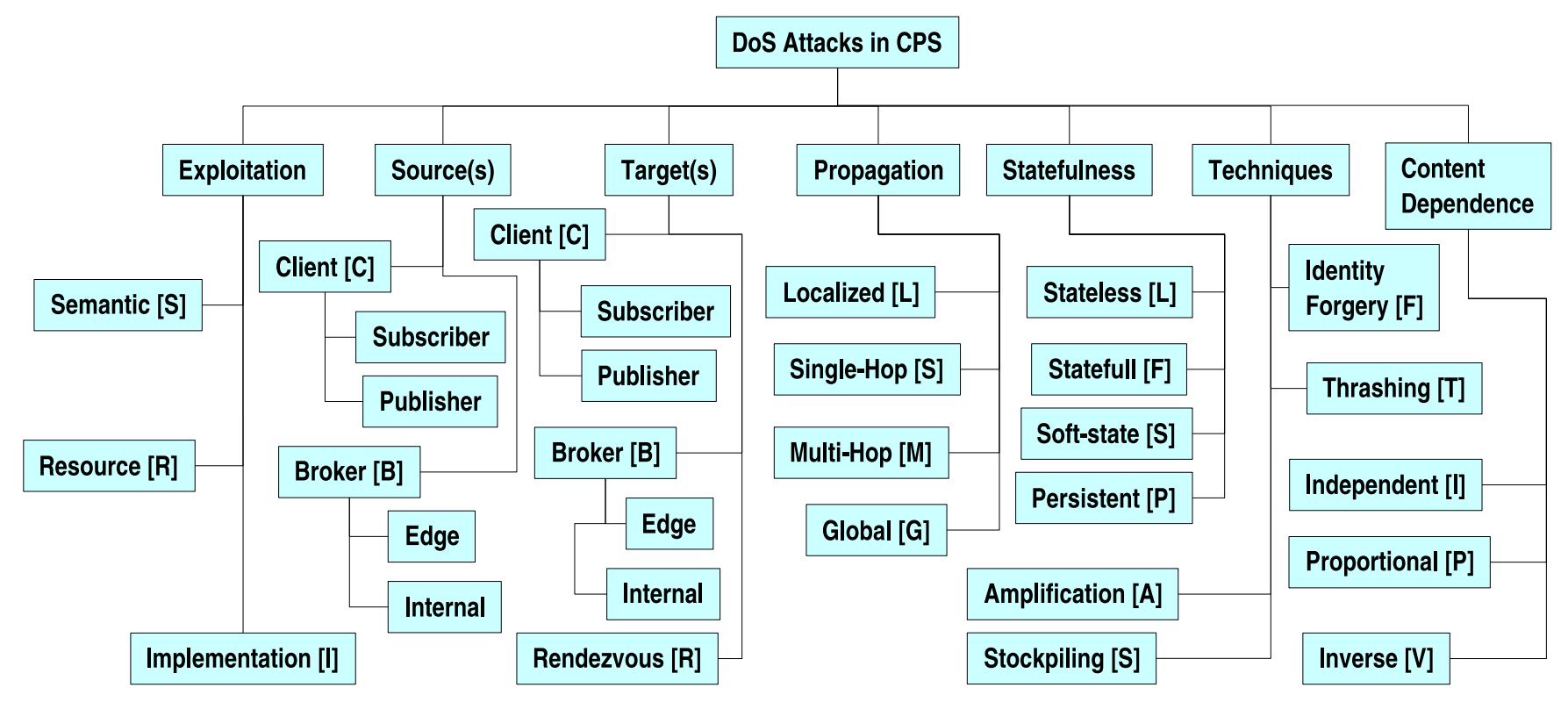

Figure 5: Taxonomy tree for Denial of Service attacks in CPS systems.

For completeness, we first observe that an attack can exploit different weaknesses in a system in order to achieve DoS. Although the end result of an attack always prevents legitimate access to system services, different exploitation types imply entirely different attack strategies by the adversary and therefore, different defence mechanisms are needed by the system.

This class is similar to the load-based versus logic-based classification widely used in existing DoS work $[12,13]$. However, we include this class because there are new implications for the exploitation of CPS systems that are not addressed in existing classifications. We propose this classification and discuss how there are new implications specific to the domain of CPS.

\section{Resource Limitations $(R)$}

The strategy of targeting resource limitations is very familiar from existing DoS attacks. However, there are unique ways for an adversary to target resource limitations in a CPS system, as we showed in our experiments. Primarily, the need to perform content-based matching and state maintenance in a CPS system leads to new DoS attack strategies for inducing abnormal network, processing, and memory overheads. Since there has been significant research in optimizing matching performance in CPS system, blindly flooding large volumes of messages may no longer be the most effective method of achieving DoS. Lower volumes of carefully engineered messages may actually be more effective in CPS systems than blind flooding.

Generally, resource attacks are the most difficult to defend against and typically involve heuristic defensive approaches.

\section{Semantic Weaknesses (S)}

Alternatively, a DoS attack may instead target semantic weaknesses that are inherent in a system due to conceptual and theoretical design flaws. These flaws can be exploited to achieve DoS without abnormally consuming resources or violating correct behaviour according to design. Semantic weaknesses typically result when systems are designed with features and functionality in mind before security and so must be dealt with by revisiting the theory behind the design itself. Most often, security is considered an orthogonal issue and a separate security layer is designed on top of the existing system. In such cases, the original system without the security layer is still considered to suffer from exploitable semantic weaknesses. Even a well-defined implementation specification can still suffer from semantic weaknesses.

For example, message covering is a common optimization technique used in CPS systems. However, there is no standard semantic for uncovering. Depending on whether uncovering occurs using a filter or per-message basis, different state maintenance overheads will be incurred. As such, the semantics of uncovering will have DoS consequences regardless of how the actual system is implemented.

\section{Implementation Flaws (I)}

Finally, a DoS attack may also target implementation flaws that are specific to a particular realization of a system. Traditionally, semantic and implementation weaknesses have been lumped together as logic-based DoS attacks but we feel that there is motivation for a more fine-grained classification. Implementation weaknesses refer to errors in implementation that can be fixed by patching the realized system without revisiting any concepts or theories. Essentially, these are implementation "bugs". Because there has yet to be a single universally accepted semantic for the CPS paradigm, systems tend to be in the prototypical stages. Therefore, it is important to distinguish DoS attacks targeting semantic weaknesses (concepts applicable to different systems using the same semantic) from implementation weaknesses (specific to a certain realization). Admittedly, the line between concept and implementation is up to a system designer unless a specification is being followed.

Using the covering example again, two systems may use the same uncovering semantic, but one may be an inefficient implementation that exaggerates the overhead of state 
maintenance.

\subsection{Attack Source(s)}

The source(s) of an attack are one or more entities under the control of an adversary. The adversary may actively send messages from these entities or use them passively to corrupt existing messages. In CPS systems, attacks can be sourced from clients, brokers, or some combination of both if multiple entities are needed. It is important to classify the entities from which an attack originates because different attacks are more suitably mounted from different sources and will again require different defence mechanisms.

Most existing DoS attacks originate from a network of distributed clients in the form of Distributed DoS (DDoS) attacks. Rarely do attacks originate from within the networking infrastructure. In CPS systems however, we cannot ignore the possibility of open or cooperative infrastructures where trusted brokers operate alongside untrusted brokers. Furthermore, CPS systems are deployed on overlay networks running at the edge of the Internet infrastructure and are therefore more vulnerable than conventional routers.

\section{Client-Sourced (C)}

In most cases, client-sourced attacks will be easier to mount than broker-sourced attacks since clients are essentially the end-users of the system and not unlikely to be less strictly regulated. That is, an adversary is more likely to be allowed legitimate client access to a CPS system than broker access. We can also reasonably expect that there will be orders of magnitude more clients than brokers and that the systems they reside on may be less secure. This means that distributed attacks relying on scale (i.e., DDoS) are more likely to be client-sourced and that there is greater potential for compromising these clients. However, attacks sourced from clients are at a disadvantage in that clients can only attack by issuing messages to the system. This implies that resource attacks based on message volume are more likely to be client-sourced.

\section{Broker-Sourced (B)}

Generally, it will be more difficult for an adversary to get malicious brokers into a system ${ }^{3}$ so CPS system designers should be more wary of client-sourced attacks in this respect. On the other hand, broker-sourced attacks (if successful) can be more severe since brokers have more control over system behaviour. In addition to attacking by issuing messages, brokers can interfere with matching and routing as well. Brokers also have access to system state information and message content that clients do not have. However, brokersourced attacks may not be on the same distributed scale as client-sourced attacks. This implies that broker-sourced attacks are more suitable for attacks with a malicious routing component, especially if colluding brokers are involved.

We expect that broker-sourced attacks will be easier to defend against than client-sourced attacks since brokers can be more strictly regulated within the system.

\subsection{Attack Target(s)}

Depending on the specific nature of a DoS attack, an adversary can target brokers, clients, or both. If an attack

\footnotetext{
${ }^{3}$ The exception to this would be a more open peer-to-peer style system allowing end-users to connect their own brokers.
}

interferes with an entity receiving messages that would normally be delivered directly to it, we say that the attack targets that entity. Note that there is a difference between the entities targeted and affected (victimized) by an attack. Typically, subscribers will always be affected by DoS attacks (since notification delivery will always be disrupted regardless of the DoS attack details) but subscribers are not always directly targeted by attacks. Because of this, we use this category to distinguish the target(s) of an attack and do not consider the victim(s).

Existing DoS attacks have generally used client resources to target servers, such as the web servers of CNN or Yahoo. To the best of our knowledge, existing DoS attacks do not isolate and target end-point clients (users) out on the Internet. However, this will likely change in the CPS domain because the system is a messaging infrastructure on top of which applications are built. Clients in the CPS domain could easily be "traditional" servers from different organizations communicating over the messaging infrastructure. For instance, the web servers of CNN and Yahoo may become clients to the CPS messaging infrastructure. Therefore, the same motivations driving adversaries to target servers with existing DoS attacks will drive them to target clients in CPS systems.

\section{Client-Targeted $(C)$}

Figure 6 shows the typical entities and messages used in a baseline CPS system. Note that although we include advertisements in our baseline model, all discussions and results in this paper are relevant to CPS systems based only on subscriptions and publications as well unless advertisements are specifically mentioned. From our definition of an attack target, there is only one way to target subscribers: by interfering with delivery of notification messages $(N)$ at the final broker to client hop. Depending on the attack, this could be achieved by intercepting the notification, crashing the subscriber itself, or even corrupting subscription state at the subscriber hosting edge broker. Targeting a client does not necessarily mean the client itself must be directly attacked. Attacking a client hosting edge broker also targets any clients hosted by it.

Notice also that in a baseline CPS system, no messages are delivered to the publisher at the CPS level so it is not possible for an attack to target publishers in sense that we have defined an attack target. Crashing a publisher prevents an edge broker from receiving publications, so such an attack is actually targeting the publisher hosting edge broker. However, if a system were to develop extensions where publishers also received messages (perhaps subscriptions or publication exceptions) then they would become possible DoS attack targets. In practice though, clients will generally take on the roles of both publisher and subscriber in an application scenario.

Targeting clients is the most direct and fine-grained method for achieving DoS since the adversary can selectively target specific clients. However, it depends on the adversary having knowledge of specific client identities or possibly network topology as well.

Client-targeted attacks are easier to defend against since we only need to secure the final hop (by our definition) assuming routing within the network is secure. 


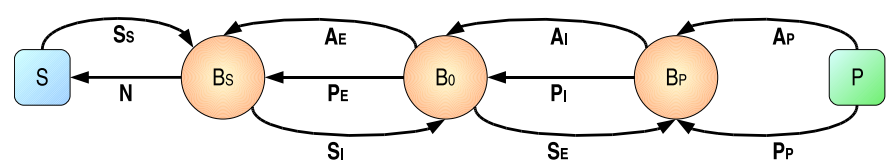

\begin{tabular}{ccc|ccc|cccc||c}
$A_{P}$ & $A_{I}$ & $A_{E}$ & $P_{P}$ & $P_{I}$ & $P_{E}$ & $S_{S}$ & $S_{I}$ & $S_{E}$ & $N$ & Target \\
\hline & & & & & & & & & $X$ & $S$ \\
& \multirow{4}{*}{$X$} & $X$ & & & $X$ & $X$ & & & & $B_{S}$ \\
& & & $X$ & & & $X$ & & & $B_{0}$ \\
& & $X$ & & & & & $X$ & & $B_{P}$
\end{tabular}

(a) The messages shown here are advertisements $\left(A_{x}\right)$, publica- (b) The target of an attack depends on which messages deliveries tions $\left(P_{x}\right)$, subscriptions $\left(S_{x}\right)$, and notifications $(N)$ being deliv- are being interfered with ered to different entities in the system

Figure 6: Classifying the target of a Denial of Service attack.

\section{Broker-Targeted (B)}

Broker-targeted attacks are much less restrictive than clienttargeted attacks. Since a broker is part of the routing infrastructure, it is possible to affect a much larger number of entities by targeting brokers. However, the adversary has less fine-grained control over which entities are attacked. This may not matter if the intended victims of an adversary are coarse-grained to begin with (an organization managing several brokers perhaps).

For example, an attack that interferes with the receipt of messages $S_{S}, A_{E}$, or $P_{E}$ by broker $B_{S}$ in Figure 6(a) is targeting the edge broker $B_{S}$ even though it ultimately interferes with notification delivery to the subscriber. Table 6(b) summarizes the entity considered to be the target of an attack depending on which message deliveries are being disrupted. In general, targeting a broker can be accomplished by intercepting messages, misrouting messages, or corrupting state. Targeting internal brokers can mean affecting their ability to perform matching or routing while attacks targeting edge brokers can additionally affect their ability to host clients or properly serve hosted clients.

Broker-targeted attacks will be more difficult to defend against since a solution must be robust under many variations of message loss during routing.

\section{Rendezvous-Targeted $(R)$}

Specifically for CPS systems built on structured overlays [15, 7,3 , we can also distinguish rendezvous points. Although rendezvous points may or may not be brokers themselves, we will use the term rendezvous point and rendezvous broker synonymously from here on. In rendezvous routing approaches, messages route towards rendezvous brokers based on the result of some hash function of the messages. Although this approach avoids the necessity for flooding advertisements, the rendezvous brokers have become the global points of reference not unlike the root DNS servers and are therefore vulnerable as targets of DoS attacks. As targets, rendezvous brokers are a special case of brokers that perform routing tasks.

Note that while rendezvous brokers exist to guarantee avoidance of false-negative notifications, a successful DoS attack is not guaranteed by successfully attacking rendezvous brokers because matching can still occur at other non-rendezvous brokers.

\subsection{Attack Propagation}

Existing DoS attacks have typically been point-to-point from malicious clients to targeted server. At most, attack packets are reflected off a third party server or router ${ }^{4}$. However, the need to replicate subscription state in CPS systems

\footnotetext{
${ }^{4}$ Although worms may cause isolated DoS effects when
}

implies that malicious messages can be distributed or routed along with legitimate messages. Since the underlying message distribution paradigm is a major distinguishing feature of CPS systems, the propagation of messages throughout a system behaves very differently depending on whether a controlled flooding [8, 5, 14], rendezvous [15, 7, 3], scoped [10], or multicast [6] paradigm is being used.

As far as we are aware, there have not been any previous efforts to classify DoS attacks according to message routing characteristics. We propose this new classification in the context of distributed CPS.

\section{Localized $(L)$}

An attack is localized if malicious messages do not propagate beyond the initial entry point broker. For instance, a basic flooding attack consisting of random publications that do not match any subscriptions would not propagate beyond the first edge broker receiving those publications. Note however, that the resulting effects of a localized attack may not be limited in the same way. In Figure 7 we illustrate a scenario in which a malicious unsubscription is injected at an edge broker hosting the publisher (at the publication source). Depending on the propagation semantics of unsubscriptions, the malicious unsubscription message may never be disseminated to any other broker so the attack is localized. However, notifications to the owner of the removed subscription will be stopped, so the effects may not be localized. Note also that DoS attacks on centralized publish/subscribe systems are necessarily localized.

\section{Single-Hop $(S)$}

In some systems, attack propagation may be restricted to a single-hop when message distribution occurs over only two brokers (the entry point broker and the next hop broker). The breadth of an attack will depend on the fan-out of a

spreading aggressively, the true attacks are typically coordinated floods from hosts compromised by the worms [11].

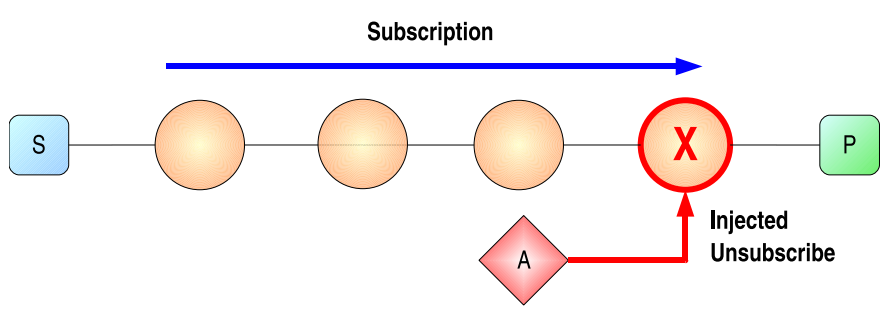

Figure 7: Localized propagation of malicious messages via unsubscription attacks. 
given broadcasted message and is equally a concern for both resource and semantic attacks.

Attack propagation may be restricted to single-hop by the distribution mechanism used. For instance, brokers in a system may leverage IP multicast rather than perform overlay routing. An attack may also be limited to single-hop due to the routing state or topology of the network. However, an attack is most likely to be restricted to a single-hop as part of some security scheme $[16,10]$.

\section{Multi-Hop (M)}

More generally, malicious messages can propagate through an arbitrary number of brokers beyond the initial entry broker in multi-hop attacks. These attacks require more care to mount than localized attacks since the filtering features of a CPS system must be avoided by the adversary.

Multi-hop propagation can be achieved by crafting attack messages that successfully match routing state. Generally, subscriptions propagate when they match advertisements and publications propagate when they match subscriptions. So attacks using subscription messages can potentially propagate back to a publishing broker, affecting all brokers along the path for instance. In this way, an adversary can use the natural propagation of messages in the system to target a publishing broker by content only. In unstructured overlay CPS systems, messages are either dropped or remain on edge brokers if no matches occur. In structured overlay CPS systems however, the propagation semantics are different due to the use of rendezvous brokers. Messages will instead propagate towards one or more rendezvous brokers unconditionally. Consequently, malicious traffic in a structured overlay CPS system cannot be localized unless the malicious traffic is injected directly at a rendezvous broker.

In addition to breadth, it is possible to further characterize specific multi-hop attack instances by depth. An attack that propagates along more hops has more depth. The potential depth of an attack will again depend on both message content and whether the CPS system is built on an unstructured or structured overlay. In structured overlays, subscriptions are guaranteed to route through a certain number of brokers en route to rendezvous broker(s) regardless of whether an intersecting advertisement exists. Since rendezvous brokers are also typically replicated, this has the effect of guaranteeing a certain propagation breadth as well.

We also note that in general, attacks using low rate traffic are expected to propagate more effectively than high rate attacks. This is because a heavily loaded broker will inherently traffic shape outbound malicious messages being propagated due to processing limitations ${ }^{5}$. The effects of a high rate attack will be more severe at the entry point of the attack, while brokers deeper in the network will be less affected by the high traffic rate. In other words, high-rate and volume attack messages are much more likely to become bottlenecked.

Also recall that multi-hop attacks are a significant danger because they can potentially transmit DoS effects to remote brokers as we demonstrated in our experiments.

\footnotetext{
${ }^{5}$ It is not unlikely that the effects of a bottlenecked application propagate down to the network layer triggering flow control mechanisms as well.
}

\section{Global $(G)$}

Finally, global attacks occur when malicious traffic is propagated to every broker in the system. Systems that support global message flooding are obviously vulnerable to these attacks, but it is also possible to mount global attacks if malicious messages are engineered to match global interests. For instance, if there is at least one subscriber at every broker subscribing to some common publication space, then a publication falling into that space would propagate to every broker in the system. CPS systems generally try to avoid global message propagation in normal situations, but we are unaware of any systems in which globally propagating attacks are impossible by design.

While a rendezvous approach avoids the need to flood messages, it introduces globally known points of reference that are themselves vulnerable as targets of DoS attacks. However, attacking all rendezvous brokers in a structured overlay does not necessarily constitute a globally propagating attack.

In theory, global attacks should be as difficult to defend as multi-hop attacks. However, this may not be the case in practice since a CPS infrastructure may span multiple organizations. Spanning multiple administrative domains will likely complicate global security mechanisms making globally propagating attacks much more difficult to defend against.

\subsection{Content Dependence}

A major feature in CPS systems is the routing of messages according to their content. Since content determines how messages are processed and delivered, an adversary can drastically change how an attack behaves by changing the content of malicious attack messages. Propagation and targeted entities are examples of attack characteristics controlled by host addresses in existing DoS attacks that are now controlled by message content in CPS systems. Whether an attack leverages content or not, and the exact nature of malicious message content being used will determine whether existing defence mechanisms are applicable.

\section{Independent (I)}

Attacks such as message dropping or malicious filter removal do not depend on the content of messages injected by an adversary. Although the adversary may choose to drop or remove certain messages, the adversary does not need to craft malicious messages with specific content to either mount the attack or make the attack more severe ${ }^{6}$.

Because no content crafting occurs on the part of the adversary, traditional security techniques based on authentication and authorization may be the most appropriate.

\section{Proportional $(P)$}

If the effectiveness of an attack depends on the adversary crafting message content, the attack can either be proportional or inversely proportional to some measure of message content complexity. For current CPS systems, the number of predicates or tuples in a message is a good reflection of message complexity. However, future CPS systems supporting filtering expressiveness beyond basic numeric and

${ }^{6}$ Malicious unsubscriptions and unadvertisements are exceptions if the CPS system uses filter-based message removal semantics rather than ID-based. 
string comparisons may need different measures of content complexity that better reflect supported filtering operations. Regardless, an attack is proportionally dependent on content complexity if the severity of the attack increases when the adversary uses malicious workloads of greater complexity. Proportional dependence is with respect to a specific characteristic of the attack, such as memory consumption or processing delay. Direct resource attacks like message flooding are likely to be proportionally dependent on workload content complexity.

Defending against content dependent attacks requires awareness of message content. However, content-based intrusion detection is a difficult problem. An accurate measure of content complexity may help predict the impact of and defend against proportionally dependent DoS attacks.

\section{Inversely Proportional ( $V)$}

An attack is inversely dependent on content complexity if the severity of the attack increases when the adversary uses malicious workloads of less complexity. At first, it may seem that all resource attacks are proportionally dependent on content complexity. However, this depends on how attacks are mounted since load can be induced by issuing highly general subscriptions, for instance. In current CPS systems, highly general subscriptions tend to attract lots of publications by having low complexity. Conversely, advertisements can typically attract more subscriptions by specifying higher complexity schemas that cover larger subscription spaces.

Again, either content awareness or an accurate measure of content complexity will help defend against such attacks.

\subsection{Statefulness of Effects}

After an adversary has stopped all direct DoS activity, it is possible that victims continue to suffer from the effects of the attack for some time. The effects of an attack may eventually dissipate or the system may have to perform some explicit recovery mechanism to rebuild correct state. The degree to which the effects of a DoS attack remain in the system gives some measure of effort needed by an adversary to mount the attack.

Except for logic-based attacks causing system crashes, existing DoS attacks are primarily stateless. However, due to the widespread maintenance of state in common CPS systems, stateful attacks beyond straight-forward system crashing may now become accessible to adversaries.

\section{Stateless $(L)$}

The effects of stateless attacks only exist while the system is actively processing malicious messages produced by an adversary. The effects of such attacks eventually disappear during the course of normal processing without the system taking special measures for recovery. Existing DoS attacks in non-CPS systems have mostly been stateless since an adversary must actively maintain DoS conditions (usually with high rate and volume malicious traffic). The lasting effects of these existing DoS attacks are typically limited to the relatively brief length of time data structures remain allocated for accepting connections and packet processing.

Similarly in CPS systems, publication messages are stateless. Although the length of time a publication spends in the system can vary significantly depending on the efficiency of matching, publications are removed from the system once processing is complete (under baseline CPS semantics). No special recovery mechanisms are required by the system. Stateless attacks are the easiest to defend against by blocking all malicious traffic.

\section{Stateful $(F)$}

On the other hand, the effects of stateful attacks remain in the system even after the processing of malicious messages produced by an adversary has been completed. Stateful attacks are an extension of traditional application level DoS attacks because the adversary can use fewer messages to cause long lasting DoS effects. However, defending against such attacks will also require explicit recovery mechanisms beyond normal message processing. There are generally two forms of state used in CPS systems, routing state and complex event detection state.

Routing state is maintained by brokers for validating, matching, and routing messages. In CPS systems, clients can modify the routing state of a broker by issuing messages. The most common messages that allow updating routing state include subscriptions, advertisements, unsubscriptions, unadvertisements, and possibly type definition or specialized update messages. These messages are candidates for enabling stateful attacks. Malicious removal of these messages (by unsubscription for example) can potentially disrupt notifications indefinitely. Malicious insertion of these messages is also a stateful attack if the performance of a system degrades with the amount of state maintained. Such corruptions of routing state must typically be repaired explicitly by the system upon detection of the attack.

Complex event detection state is maintained by brokers for correlating publications against stateful subscriptions. That is, subscriptions themselves may be stateful. In systems supporting complex event detection, publications are also candidates for mounting stateful attacks. Note that depending on the design of such a system, it may or may not be necessary to actually retain publications in the system.

Stateful attacks are more difficult to defend against since additional explicit recovery techniques are required.

\section{Soft-state $(S)$}

In between stateless and stateful attacks, the effects of softstate attacks also remain in the system after malicious messages have been processed. However, such effects are eventually removed automatically via some expiry mechanism. With respect to routing state, expiry mechanisms are typically used for fault tolerance purposes. In systems supporting complex event detection, expiry mechanisms are used to limit or window the amount of state maintained and prevent publication state from accumulating indefinitely.

An attack that is stateful against one system may only be soft-stateful in another due to differences in how state is maintained. Since soft states imply the need for continual state refreshing, we expect that state expiration will typically not occur too frequently. When state expiry is significantly infrequent, soft-state attacks become very similar to stateful attacks.

\section{Persistent $(P)$}

A CPS system may also support subscribing to historic data or recovering state from persistent storage to achieve fault tolerance. In such systems, there is an opportunity for corrupting or inserting malicious messages into persistent storage. When retrieved, these malicious messages can poten- 
tially cause DoS effects as well. Attacks are considered persistent if its effects can be triggered by retrieving malicious data off persistent storage.

\subsection{Component Techniques}

When adversaries discover effective and portable techniques for mounting DoS attacks, they will be reused in a variety of different attacks. Buffer overflows, packet reflection, and IP spoofing are examples of existing reusable techniques for mounting DoS attacks in non-CPS systems that are not attacks by themselves. Defending against these techniques is typically difficult because they are very generic, but would effectively defend against entire classes of DoS attacks. For CPS systems, we identify identity forgery, thrashing, amplification, and stockpiling techniques.

The techniques we identify in this class are not mutually exclusive since a single DoS attacks may use multiple techniques.

\section{Identity Forgery $(F)$}

In distributed CPS systems, there are generally two identities associated with each message: the identity of the original message source and the identity of the entity actually delivering the message. An adversary may forge either or both of these identities in an attack.

The identity of the original message source defines the message owner, which is used to associate messages with some entity for the purposes of state management and notification delivery at the final hop. Forging the identity of the original message owner can be used to manipulate existing state belonging to another entity or to inject arbitrary messages on behalf of another entity.

The identity of the entity actually delivering the message is used for reverse path forwarding. Forging this identity will allow an adversary to cause misrouting of messages. For instance, notifications could be directed to some victim client. While this second type of identity forgery is similar in principle to IP spoofing used in traditional DoS attacks, the effects are more restricted in CPS systems. IP spoofing is often used to reflect packets at a victim and can target any system with a routable IP address by forging the source identity of the victim. However, this is not the case in distributed CPS systems where routing depends on state, message content, and only the previous routing hop. Identity forgery can at most misroute messages amongst the common neighbours of a broker which limits the applicability of traditional reflection attacks in CPS systems.

\section{Thrashing (T)}

Thrashing consumes resources in the system by causing rapid and frequent state changes (analogous to thrashing in memory management). Thrashing is a special case of more general resource attacks so its effectiveness depends on how much system load can be generated in terms of processing and network traffic.

Unlike straight forward message flooding attacks, the goal is to induce load by abusing repeated state changes that are processing intensive. This can be accomplished using a set of messages that will likely include unsubscriptions (or unadvertisements). Thrashing can succeed where flooding fails by avoiding optimization schemes that typically depend on "normal" message patterns. Generally, this will result in pathological state maintenance behaviour. This technique is most useful in systems with covering and tight controls over messages issued by a client (for instance, when there is little flexibility in building a large number of unique filters for flooding).

\section{Amplification (A)}

Amplification gives an adversary the ability to induce delivery of multiple messages to a single entity by injecting a single malicious message. Note that amplification does not include multicasting which only delivers a single message to multiple distinct entities.

Several characteristics of the CPS paradigm are prone to amplification. For instance, a new advertisement may attract many dormant subscriptions or an unsubscription may trigger forwarding of multiple subscriptions. Existing DoS attacks such as reflection use amplification by leveraging packet re-transmissions to spoofed IP addresses. However, packet re-transmissions have a fixed amplification factor (fixed number of re-transmissions). In contrast, existing CPS systems currently have features that potentially allow arbitrarily high amplification factors. We discuss amplification in the context of specific attacks in more detail later.

\section{Stockpiling $(S)$}

To be successful, some attacks may depend on certain state configurations in the system. Because of this, an adversary may first need to setup the state needed to mount an attack. Such malicious traffic will need to be stateful but is not technically part of the attack because it causes no DoS effects by itself. If the malicious state build up is discovered and dealt with, the system can avoid the DoS attack altogether. We use the term stockpiling to describe such "attacks" that build up malicious state in preparation for or to enable the true DoS attack (which itself may or may not be stateful). For instance, an adversary could first inject a number of malicious advertisements or subscriptions into the system that significantly increases the effects of a subsequent publication flooding attack. We elaborate more on the use of stockpiling in specific attacks in Section 5.

Vulnerability to stockpiling is introduced by allowing system users to issue messages that remain in the system. CPS systems have so far assumed voluntary removal of such state by the system users themselves, but little work has been done to investigate how a system can deal with uncooperative users such as those intending to mount DoS attacks. Access control schemes are a possible prevention approach but do not address situations where an adversary is able to circumvent the access control layer or compromises an entity with legitimate access. Note that the use of soft states does not prevent stockpiling since an adversary can easily refresh any stockpiled state.

\section{DISCUSSION}

In Section 4, we noted that like recently emerging application level DoS attacks, an adversary can attack a CPS system using relatively low volumes of malicious messages if the messages are crafted carefully. Blindly flooding large volumes of messages is not necessarily the most effective way of attacking a CPS system. As shown by our experiments in Section 3, the statefulness of CPS systems is a key exploitable vulnerability that can be leveraged by advanced DoS attacks. In this section, we elaborate on our experimental results and illustrate the significance of the classification 


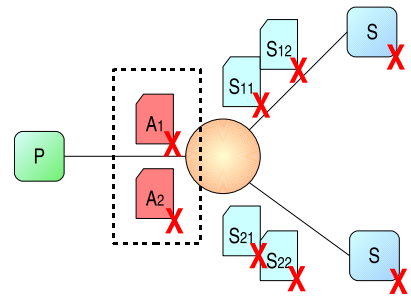

(a) Filter-based unadvertisement

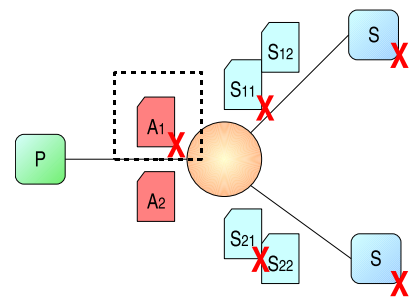

(b) ID-based unadvertisement

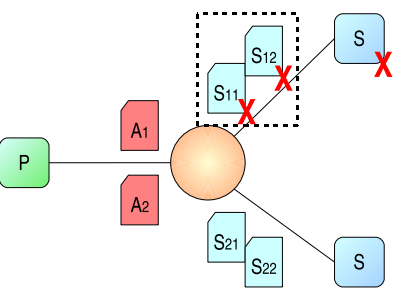

(c) Filter-based unsubscription

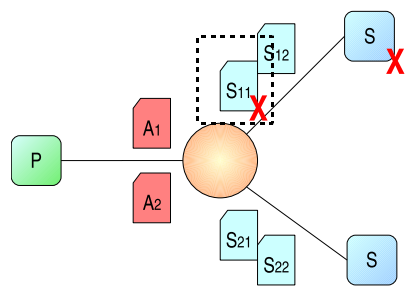

(d) ID-based unsubscription

Figure 8: Different filter removal semantics can significantly change how notification delivery is affected across advertisement and subscription spaces. The filters and clients that can be affected by a single filter removal operation (dotted box) under each semantic are marked by an $X$.

used in our taxonomy by discussing stateful DoS attacks in more detail.

First, since advertisements and subscriptions are used to establish routing paths, they can be injected by an adversary to control the flow of messages through the system. Highly generic subscriptions can be used to attract large amounts of publication traffic to the adversary-hosting broker or redirect large amounts of notification traffic to a client hosted by the same broker. Similarly, highly generic advertisements can be used to attract large numbers of subscriptions. Advertisement injections of this form consume more state than subscription injections by attracting subscription messages, but may attract fewer overall messages if the assumption that legitimate publication traffic exceeds legitimate subscription traffic holds true. Additionally, even if the adversary does choose to inject malicious advertisements or subscriptions to load the system directly, the adversary can still "build-up" a significant amount of malicious state stealthily before injecting a final burst of malicious messages to complete the actual attack. This two-phase "build-up"/flood approach allows an adversary to weaken the resilience of brokers deeper in the network with stealthy low-rate messages that are minimally affected by the traffic shaping effects of network bottlenecks. CPS researchers have informally expressed concerns over the effects of highly generic filters and malicious state before, but it is not until now that we are able to classify such attacks as using the stockpiling technique as presented in our taxonomy. This shows that our taxonomy is indeed useful for structuring DoS in CPS systems with respect to real concerns and actual attack instances.

Second, since advertisement and subscription state needs to be maintained in order for messages to be delivered, it is also possible to mount DoS attacks by maliciously removing advertisements or subscriptions owned by another entity. However, the semantics of malicious state removal attacks are not as straight-forward as they first appear. This is because there are currently two known semantics for unadvertisements (and similarly for unsubscriptions): ID-based and filter-based. In some systems, filter removal is based on ID. That is, a single unadvertisement (unsubscription) corresponds to the removal of a single advertisement (subscription). However, in some other systems [8], filter removal is done using another filter that covers one or more filters to be removed and the "unfilter" is expressed using normal filter syntax. That is, a single unadvertisement (unsubscription) may remove multiple advertisements (subscriptions). The filter-based removal semantics result in potentially broader attacks since a single injected unfilter can potentially cover and remove multiple legitimate filters. The effect of malicious filter removal is to cut off the flow of publications, and possibly also trigger state maintenance in the case of unadvertisements. Figure 8 illustrates the differences between unadvertisement and unsubscription injections under the ID-based and filter-based semantics we described. There is currently no consensus on whether ID-based or filter-based filter removals should be used in CPS. However, as we show here, there are significant DoS implications arising from unfilter semantics that are independent of actual implementation. Since this concern is classified under semantic weaknesses in our taxonomy, it again shows that our taxonomy is useful for classifying real concerns and actual attack instances.

\section{RELATED WORK}

Mirkovic and Reiher [12] develop a taxonomy specifically for Distributed DoS attacks in which an adversary controls multiple attacking entities. However, their taxonomy is specific to DDoS attacks in non-CPS systems. Our taxonomy supplements their work both by identifying classifications relevant in the CPS domain and by not restricting ourselves to only DDoS attacks.

In the CPS domain, there has been relatively little focus on DoS attacks, which are generally grouped together with other general security concerns.

Srivatsa and Liu [16] propose a security system on top of publish/subscribe based on layers of software guards. Their authentication mechanisms successfully limit the effects of message injection attacks to a single-hop. However, their work is focused on providing a general security solution and does not discuss DoS attacks in-depth.

Wang et al. [17] discussed several broad security issues and requirements including DoS. They briefly discuss three potential approaches for defending against DoS attacks: publication limits, CPU-cycle-based payments, and challengebased authentication. However, they also do not discuss the effects of DoS attacks in any detail.

To the best of our knowledge, our work is the first to conduct a detailed study of DoS effects and highlight key DoS concerns new to CPS systems. As far as we are aware, we are also the first to provide a taxonomy of DoS attack characteristics in CPS systems, structuring a previously vague area. 


\section{CONCLUSION}

In this paper, we have presented experimental results that highlight some key DoS concerns arising in CPS systems. In our experiments, we have found that message propagation, content complexity, and state maintenance are characteristics introduced by the CPS paradigm with new DoS implications. The effects of resource attacks depend heavily on how these characteristics are leveraged by adversaries. In particular, load localization effects can be used as a DoS resilience technique, while remotely induced DoS effects are a significant concern since such attacks will be difficult to defend against. Furthermore, both the content complexity and statefulness of malicious workloads will significantly affect the severity of load-based DoS attacks. We believe this will be a general concern for many CPS systems. Accurately estimating the content complexity of messages and effectively controlling malicious message state are two directions of research that we are pursuing as future work.

Based on our experimental results, we have also proposed a taxonomy for classifying DoS attack characteristics and concerns in the domain of CPS systems. Our taxonomy is complete in the sense that it can be used to easily classify both existing and future DoS attack instances in this domain. Our taxonomy also facilitates comparing the inherent DoS resilience of different CPS system designs as well as comparing the effectiveness of different defence mechanisms.

Finally, we have discussed several DoS attack instances in detail with respect to our experimental results and taxonomy. Our discussion provides further insight into the implications of state maintenance on DoS vulnerabilities. In particular, message stockpiling and malicious unfiltering are two fundamental vulnerabilities that can dramatically increase the effects of DoS attacks and should be considered by DoS resilient CPS systems.

It is our hope that this paper will stimulate discussion about DoS attacks and defences in both existing and future CPS systems.

\section{Acknowledgements}

This research was funded in part by OCE, NSERC, CA and Sun. We would also like to thank Vinod Muthusamy and the entire Middleware Systems Research Group at the University of Toronto for their valuable feedback regarding this work.

\section{REFERENCES}

[1] [2]

http://www.cisco.com/en/US/products/ps6480/index.html.

http://www.solacesystems.com/products/Tech_leadership.asp.

[3] I. Aekaterinidis and P. Triantafillou. PastryStrings: A Comprehensive Content-Based Publish/Subscribe DHT Network. In ICDCS, 2006.

[4] M. K. Aguilera, R. E. Strom, D. C. Sturman, M. Astley, and T. D. Chandra. Matching Events in a Content-based Subscription System. In Principles of Distributed Computing, 1999.

[5] G. Banavar, T. Chandra, B. Mukherjee, J. Nagarajarao, R. E. Strom, and D. C. Sturman. An Efficient Multicast Protocol for Content-based Publish-Subscribe Systems. In International Conference on Distributed Computing Systems, 1999.

[6] F. Cao and J. Singh. MEDYM: Match-Early and Dynamic Multicast for Content-base Publish/Subscribe Service Networks. In IEEE International Conference on Distributed Computing Systems Workshops, 2005.
[7] N. Carvalho, F. Araujo, and L. Rodrigues. Reducing Latency in Rendezvous-Based Publish-Subscribe Systems for Wireless Ad Hoc Networks. In ICDCSW, 2006.

[8] A. Carzaniga, D. Rosenblum, and A. Wolf. Design and Evaluation of a Wide-Area Event Notification Service. ACM Transactions on Computer Systems, 19(3):332-383, 2001.

[9] F. Fabret, H. A. Jacobsen, F. Llirbat, J. Pereira, K. A. Ross, and D. Shasha. Filtering Algorithms and Implementation for Very Fast Publish/Subscribe Systems. In Sigmod, 2001.

[10] L. Fiege, M. Mezini, G. Muhl, and A. P. Buchmann. Engineering Event-Based Systems with Scopes. In ECOOP, 2002.

[11] K. J. Houle, G. M. Weaver, N. Long, and R. Thomas. Trends in Denial of Service Attack Technology. Technical report, CERT Coordination Center, 2001.

[12] J. Mirkovic and P. Reiher. A Taxonomy of DDoS Attack and DDoS Defense Mechanisms. ACM SIGCOMM Computer Communications Review, 34 (2):39-54, 2004.

[13] D. Moore, C. Shannon, D. J. Brown, G. M. Voelker, and S. Savage. Inferring Internet Denial-of-Service Activity. ACM Transactions on Computer Systems, 24 (2):115-139, 2006.

[14] G. Muhl. Large-Scale Content-Based Publish/Subscribe Systems. PhD thesis, Vom Fachbereich Informatik der Technischen Universitat Darmstadt, 2002.

[15] P. R. Pietzuch and J. M. Bacon. Hermes: A Distributed Event-Based Middleware Architecture. In DEBS, 2002.

[16] M. Srivatsa and L. Liu. Securing Publish-Subscribe Overlay Services with EventGuard. In 12th ACM conference on Computer and communications security, 2005.

[17] C. Wang, A. Carzaniga, D. Evans, and A. Wolf. Security Issues and Requirements for Internet-Scale Publish-Subscribe Systems. In Hawaii International Conference on System Sciences, 2002. 